\title{
Dimerization of Oleate in Aqueous Solutions
}

\author{
P. SOMASUNDARAN, K. P. ANANTHAPADMANABHAN, AND I. B. IVANOV ${ }^{1}$ \\ School of Engineering and Applied Science, Columbia University, New York, New York 10027
}

Received July 28, 1983; accepted October 6, 1983

\begin{abstract}
Surfactants have been considered by a number of investigators to form premicellar multimers such as dimers and acid-soap. In this paper a method based upon a thermodynamic model for the dependence of surface tension on surfactant concentration to identify premicellar aggregation is presented. Surface tension data for potassium oleate solutions is analyzed using the above model and the deviations in surface tension behavior from that expected of monomeric oleate is attributed to dimerization. The formation constants for doubly charged oleate dimer is estimated from this analysis to be $6 \times 10^{3}$ $\left(\mathrm{kmol} / \mathrm{m}^{3}\right)^{-1}$.
\end{abstract}

\section{INTRODUCTION}

Surfactants are normally believed to exist as monomers up to a certain concentration and to aggregate to form micelles or to precipitate above that concentration. However, a number of recent investigations (1-22) have indicated the formation of low-molecular weight aggregates such as dimers and ionomolecular complexes in surfactant solutions. Evidence for the formation of premicellar aggregates have come from studies on surfactant solution properties such as conductivity (710), molar volume (13-15), osmotic coefficient $(10,16,17)$, transport number $(8)$, hydrolysis $(3,4,6,12,21,22)$, and surfactant partitioning between an aqueous and organic phase (11, 23). In most of these studies doubly charged dimers have been considered to be a dominant form of the premicellar aggregate.

The major driving force for the associative interactions in surfactant solutions can be considered to be the "hydrophobic" bonding between the nonpolar parts of the surfactant species. On the basis of the postulate that water molecules are more ordered near a hydrocarbon chain than in bulk (24) association of

\footnotetext{
${ }^{1}$ Present address: Department of Physical Chemistry, University of Sofia, Bulgaria.
}

monomers can be expected to decrease the number of water molecules in such an ordered state. Indeed, the resultant increase in entropy will favor the formation of multimers. On the other hand, interactions of polar and/or ionic heads can lead to a positive or negative contribution depending upon the nature of the species involved in association. Formation of doubly charged dimers, for example, will have contributions arising from the charge/charge repulsion which will oppose the formation of dimers.

There have been attempts to theoretically estimate the free energy changes involved in hydrophobic bonding and dimerization (16, 25-27). Bangs (16), for example, considered the free energy of formation of dimers, $\Delta G^{\circ}{ }_{\mathrm{d}}$, to be a summation of the chain-chain (hydrophobic), electrostatic, and kinetic energy changes involved in dimerization. Bangs equated the chain-chain interaction energy to the change in hydrocarbon/water interfacial energy involved in dimerization and calculated the latter from the oil/water interfacial tension and the difference between the interfacial areas of a dimer and that of two monomers. The estimation of electrostatic energy, on the other hand, involves the dielectric constant of the medium. Bangs (16) used a value of 78 , the dielectric constant of bulk water in his cal- 
culations. Since water molecules are more ordered near the hydrocarbons and ions than in the bulk, dielectric constant in the immediate vicinity of surfactant ion will be lower than 78. The marked dependency of calculated $K_{\mathrm{d}}\left(=\exp \left(-\Delta G^{\circ}{ }_{\mathrm{d}} / R T\right)\right)$ on the dielectric constant can be seen from Fig. 1. The uncertainties in the value of dielectric constant to be used in the calculations thus makes the theoretical predictions less reliable.

In contrast to the above, a number of reports in the literature question the formation of such association complexes (28-33). Potentiometric measurements of van Voorst Vader (29), for example, showed no significant evidence for association in dodecylsulfate solutions. The EMF data of Kolthoff and Johnson (31) for potassium laurate and of Malik and co-workers (32) for potassium laurate, myristate, and palmitate exhibited Nernstian response indicative of the monomeric characteristic of these surfactants. Possible poisoning of the elec-

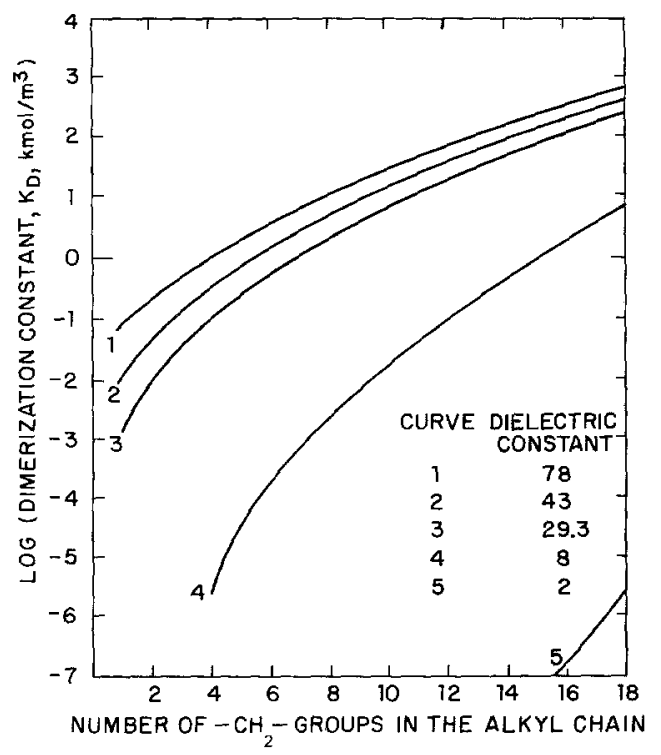

FiG. 1. $K_{\mathrm{d}}$ vs chain length for different values of dielectric constant. $K_{\mathrm{d}}=\exp \left(-\Delta G^{\circ}{ }_{\mathrm{d}} / R T\right), \Delta G_{\mathrm{d}}^{\circ}=\Delta G^{\circ}{ }_{\mathrm{c}-\mathrm{c}}$ $+\Delta G_{\text {elec }}^{\circ}+\Delta G^{\circ}{ }_{\mathrm{KE}}, \Delta G^{\circ}{ }_{\mathrm{c}-\mathrm{c}}$, and $\Delta G_{\mathrm{KE}}^{\circ}$ used in computing this diagram are from Bangs' (16) estimates. The dielectric constants used are (1) 78, water; (2) 43, average of water and ice; (3) 29.3, average of ice, water, and hydrocarbon oil; (4) 8, ice; and (5) 2, hydrocarbon oil. trodes due to surfactant adsorption on electrodes and effects of liquid junction potentials are however to be taken into account while using the potentiometric results.

Rijnbout (33) based on his surface tension data for hexadecyl-trimethyl-ammonium bromide solutions argued against any association in the premicellar region. It is, however, to be noted that surface tension results, as will be evident from the discussion in a later section, can indicate associative interactions only if the solution conditions are properly chosen and if results are obtained over a wide concentration range. Also the Wilhelmy plate technique used by Rijnbout for measurements of surface tension of amine solutions was using platinum which is known to get poisoned in amine solutions. Adsorption of amine on the sensor plate can lead to severe contact angle problems and thus can result in erroneous surface tension values (34).

Evidently much uncertainty remains on the question of premicellar association and since an accurate knowledge of the phenomenon is important in developing also a full understanding of various interfacial processes such as flotation, a systematic investigation of the formation of premicellar aggregates in a typical surfactant, potassium oleate was made in this study.

\section{A THERMODYNAMIC MODEL FOR SURFACE TENSION OF SURFACTANT SOLUTIONS CONTAINING PREMICELLAR COMPLEXES}

In this work the surface tension behavior of potassium oleate solutions was studied to investigate the premicellar behavior of this surfactant, since this property is very sensitive to changes in the activity of surfactant species in the premicellar region. Toward this purpose, a thermodynamic model taking into account the formation of surfactant dimers and acidsoap for the dependence of surface tension on surfactant concentration was first developed.

Consider an anionic surfactant $\mathrm{RH}$ in an aqueous solution in the presence of an indifferent electrolyte MX. Species to be considered in this system are: $\mathbf{R H}, \mathbf{R}^{-}, \mathbf{R}_{2} \mathbf{H}^{-}, \mathbf{R}_{2}^{2-}, \mathbf{M}^{+}$, 
$\mathrm{X}^{-}, \mathrm{H}_{2} \mathrm{O}, \mathrm{H}^{+}$, and $\mathrm{OH}^{-}$. Multimers such as trimers and tetramers have not been considered in this treatment at this stage, because of the reduced probability of their formation resulting from the increased electrostatic repulsion between charged heads which must now be placed closer to one another. If necessary, higher multimers can, however, be easily included in this treatment. The following bulk chemical equilibria and the corresponding equilibrium constants can be written for this system:

$$
\begin{aligned}
& \mathrm{RH}_{l} \rightleftarrows \mathrm{RH} \\
& K_{\mathrm{sol}}=\frac{\{\mathrm{RH}\}^{-}}{\left\{\mathrm{RH}_{l}\right\}}, \\
& \mathrm{RH} \rightleftarrows \mathrm{R}^{-}+\mathrm{H}^{+} \\
& K_{\mathrm{a}}=\frac{\left\{\mathrm{R}^{-}\right\}\left\{\mathrm{H}^{+}\right\}}{\{\mathrm{RH}\}}, \\
& 2 \mathrm{R}^{-} \rightleftarrows \mathrm{R}_{2}^{2-} \\
& K_{\mathrm{d}}=\frac{\left\{\mathbf{R}_{2}^{2-}\right\}}{\left\{\mathbf{R}^{-}\right\}^{2}} \text {, } \\
& \mathbf{R}^{-}+\mathbf{R H} \rightleftarrows \mathbf{R}_{2} \mathbf{H}^{-} \quad K_{\text {ad }}=\frac{\left\{\mathbf{R}_{2} \mathrm{H}^{-}\right\}}{\left\{\mathbf{R}^{-}\right\}\{\mathbf{R H}\}}, \\
& \mathrm{H}_{2} \mathrm{O} \rightleftarrows \mathrm{H}^{+}+\mathrm{OH}^{-}, \\
& \mathrm{MX} \rightleftarrows \mathrm{M}^{+}+\mathrm{X}^{-},
\end{aligned}
$$

where terms in the brackets indicate the activities of the respective species. The Gibbs equation for adsorption of various species in this system can be written as

$$
-\partial \gamma=\sum_{i} \Gamma_{i} \partial \mu_{i}
$$

where $\gamma$ is the surface tension, $\Gamma_{i}$ and $\mu_{i}$ are Gibbs excess and the chemical potential of the species $i$, respectively. The Gibbs equation for this system with the standard convention $\Gamma_{\mathrm{H}_{2} \mathrm{O}}=0$ is

$$
\begin{aligned}
-\partial \gamma= & \Gamma_{\mathrm{RH}} \cdot \partial \mu_{\mathrm{RH}}+\Gamma_{\mathrm{R}^{-}} \cdot \partial \mu_{\mathrm{R}^{-}} \\
& +\Gamma_{\mathrm{R}_{2} \mathrm{H}^{-}} \cdot \partial \mu_{\mathrm{R}_{2} \mathrm{H}^{-}}+\Gamma_{\mathrm{R}_{2}^{2-}} \partial \mu_{\mathrm{R}_{2}^{2-}} \\
& +\Gamma_{\mathrm{H}^{+}} \cdot \partial \mu_{\mathrm{H}^{+}}+\Gamma_{\mathrm{OH}^{-}} \cdot \partial \mu_{\mathrm{OH}^{-}} \\
& \quad+\Gamma_{\mathrm{M}^{+}} \cdot \partial \mu_{\mathrm{M}^{+}}+\Gamma_{\mathrm{X}^{-}} \cdot \partial \mu_{\mathrm{X}^{-}}
\end{aligned}
$$

Using the various bulk equilibria relations such as

$$
\partial \mu_{\mathrm{RH}}=\partial \mu_{\mathrm{R}^{-}}+\partial \mu_{\mathrm{H}^{+}}
$$

and assuming that the chemical potential of the solvent does not change appreciably Eq. [8] can be modified to yield

$$
\begin{aligned}
-\partial \gamma= & \left\{\Gamma_{\mathrm{RH}}+2 \Gamma_{\mathrm{R}_{2} \mathrm{H}^{-}}+2 \Gamma_{\mathrm{R}_{2}^{2-}}+\Gamma_{\mathrm{R}^{-}}\right\} \partial \mu_{\mathrm{RH}} \\
+\{ & \left.\Gamma_{\mathrm{H}^{+}}-\Gamma_{\mathrm{R}_{2} \mathrm{H}^{-}}-2 \Gamma_{\mathrm{R}_{2}^{2-}}-\Gamma_{\mathrm{R}^{-}}-\Gamma_{\mathrm{OH}^{-}}\right\} \\
& \times \partial \mu_{\mathrm{H}^{+}}+\Gamma_{\mathrm{M}^{+}} \partial \mu_{\mathrm{M}^{+}}+\Gamma_{\mathrm{X}^{-}} \partial \mu_{\mathrm{X}^{-}} . \quad[9]
\end{aligned}
$$

This equation involves the chemical potential of only a single surfactant species, namely, RH. Since it is the total surfactant concentration, rather than the activity of any single species that is easily known, it will be convenient to rewrite $\partial \mu_{\mathrm{RH}}$ in the above equation in terms of the total surfactant concentration. This can be done as follows:

$$
\partial \mu_{\mathrm{RH}}=R T \partial \ln c_{\mathrm{RH}}+R T \partial \ln f,
$$

where $f$ is the activity coefficient of $R H$. The term $\partial \ln c_{\mathrm{RH}}$ can be related to $c_{\mathrm{T}}$ the total surfactant concentration in the system by considering the mass balance in the bulk,

$$
c_{\mathrm{T}}=c_{\mathrm{RH}}+2 c_{\mathrm{R}_{2} \mathrm{H}^{-}}+2 c_{\mathrm{R}_{2}^{2-}}+c_{\mathrm{R}^{-}}
$$

where $c_{i}$ is the concentration of species $i$ in the bulk. It is further assumed that the adsorption at the liquid/air interface does not affect the bulk concentration significantly. Also if the activity coefficients of various surfactant species in dilute solutions is assumed to be unity, one can obtain

$$
c_{\mathrm{RH}}=\frac{-\left(1+K_{\mathrm{H}}\right)+\sqrt{\left(1+K_{\mathrm{H}}\right)^{2}+8 z c_{\mathrm{T}}}}{4 z},
$$

where

and

$$
z=K_{\mathrm{ad}} K_{\mathrm{H}}+K_{\mathrm{d}} K_{\mathrm{H}}^{2}
$$

$$
K_{\mathrm{H}}=K_{\mathrm{a}} / c_{\mathrm{H}^{+}} .
$$

Equation [12] can be differentiated to obtain

$$
\partial \ln c_{\mathrm{RH}}=\alpha \partial \ln c_{\mathrm{T}}+\beta \partial \ln c_{\mathrm{H}^{+}},
$$

where $\alpha$ and $\beta$ are given by

$$
\alpha=\frac{1}{2}+\frac{1}{2}\left\{\frac{1+K_{\mathrm{H}}}{\sqrt{\left(1+K_{\mathrm{H}}\right)^{2}+8 z c_{\mathrm{T}}}}\right\}
$$




$$
\begin{aligned}
\beta= & \frac{1}{2}-\frac{1}{2}\left[\frac{1-K_{\mathrm{H}}}{\left(\left(1+K_{\mathrm{H}}\right)^{2}+8 z c_{\mathrm{T}}\right)^{1 / 2}}\right]+K_{\mathrm{d}} K_{\mathrm{H}}^{2} \\
& \times\left(\frac{1}{2 z}-\frac{1+K_{\mathrm{H}}}{\left(\left(1+K_{\mathrm{H}}\right)^{2}+8 z c_{\mathrm{T}}\right)^{1 / 2} 2 z}\right) .
\end{aligned}
$$

Substituting for $\partial \ln c_{\mathrm{RH}}$ in Eq. [10] the following equation can be obtained for change in surface tension with surfactant concentration and $\mathrm{pH}$ :

$$
\begin{aligned}
& -\frac{\partial \gamma}{R T}=\Gamma_{\mathrm{T}} \alpha \partial \ln c_{\mathrm{T}}+\Gamma_{\mathrm{T}} \beta \partial \ln c_{\mathrm{H}} \\
& +\left\{\Gamma_{\mathrm{H}^{+}}-\Gamma_{\mathrm{R}^{-}}-\Gamma_{\mathrm{R}_{2} \mathrm{H}^{-}}-2 \Gamma_{\mathrm{R}_{2}^{2-}}-\Gamma_{\mathrm{OH}^{-}}\right\} \\
& \quad \times \partial \ln c_{\mathrm{H}}+\Gamma_{\mathrm{M}^{+}} \partial \ln c_{\mathrm{M}^{+}} \\
& \quad+\Gamma_{\mathrm{X}^{-}} \partial \ln c_{\mathrm{X}^{-}}, \quad
\end{aligned}
$$

where

$$
\Gamma_{\mathrm{T}}=\Gamma_{\mathrm{RH}^{-}}+\Gamma_{\mathrm{R}^{-}}+2 \Gamma_{\mathrm{R}_{2} \mathrm{H}^{-}}+2 \Gamma_{\mathrm{R}_{2}^{2-}}
$$

coefficients $\alpha$ and $\beta$ account for all the associative interactions in solution. The value of $\alpha$ lies between 1 and $1 / 2$, the former corresponding to the state of total dissociation and latter to that of complete association. Similarly the value of $\beta$ for the case where RH and $\mathrm{R}^{-}$ are the only surfactant species in the system is given by

$$
\beta=K_{\mathrm{H}} /\left(1+K_{\mathrm{H}}\right) .
$$

Equation [18] is not in a form that is convenient for use for the present purpose, but can be simplified for specific solution conditions. Under high pH conditions ( $>11)$, for example, the equations can be simplified as follows.

The term $z$ (see Eq. [13]) can be written as

$$
z=K_{\mathrm{d}} K_{\mathrm{H}}^{2}\left\{1+K_{\mathrm{ad}} /\left(K_{\mathrm{d}} K_{\mathrm{H}}\right)\right\} .
$$

The above equation can be further simplified, provided an estimate of the second term in the brackets can be made. Even though the values of $K_{\mathrm{ad}}$ and $K_{\mathrm{d}}$ are not known, the ratio of $K_{\mathrm{ad}} / K_{\mathrm{d}}$ can be estimated as

$-R T \ln K_{\mathrm{d}}$

$$
=\Delta G^{\circ}{ }_{\mathrm{c}-\mathrm{c}}+\Delta G_{\mathrm{KE}}^{\circ}+\Delta G_{\text {elec }}^{\circ},
$$

$-R T \ln K_{\mathrm{ad}}$

$$
=\Delta G^{\circ}{ }_{\mathrm{c}-\mathrm{C}}+\Delta G^{\circ}{ }_{\mathrm{KE}}+\Delta G_{\mathrm{H}}^{\mathrm{o}},
$$

where $\Delta G^{\circ}{ }^{\circ}-\mathrm{c}=$ free energy change due to hydrocarbon chain-chain interaction, $\Delta G^{\circ}{ }_{\mathrm{KE}}=$ kinetic energy change, $\Delta G^{\circ}$ elec $=$ electrostatic energy involved in the formation of doubly charged dimer, and $\Delta G^{\circ}{ }_{\mathrm{H}}=$ hydrogen bonding energy involved in the formation of acid soap. Assuming that $\Delta G^{\circ}{ }^{\circ}-\mathrm{c}$ and $\Delta K^{\circ}{ }_{\mathrm{KE}}$ involved in the formation of both doubly charged dimer and acid-soap are same, it can be shown that

$R T \ln \left(\left(K_{\mathrm{ad}}\right) /\left(K_{\mathrm{d}}\right)\right)=\Delta G^{\circ}{ }_{\text {elec }}-\Delta G^{\mathrm{o}}{ }_{\mathrm{H}}$.

Using reasonable estimates of $\Delta G^{\circ}$ clec and $\Delta G^{\circ}{ }_{H}(35)$, it can be shown that the value of $K_{\mathrm{ad}} / K_{\mathrm{d}}$ can be as high as $10^{4}$. Since $K_{\mathrm{a}} \cong 10^{-5}$ for a weakly anionic surfactant, Eq. [21] for $\mathrm{pH}$ values 11 and above can be simplified to read

and

$$
\alpha=\frac{1}{2}+\frac{1}{2} \cdot \frac{1}{\left(1+8 K_{\mathrm{d}} c_{\mathrm{T}}\right)^{1 / 2}}
$$

$$
\beta=1 \text {. }
$$

The dependence of surface tension on surfactant concentration under constant high $\mathbf{p H}$ $(>11)$ and high ionic strength conditions can now be written as

$$
\begin{aligned}
& -\frac{\partial \gamma}{R T \partial \ln c_{\mathrm{T}}} \\
& \quad=\Gamma_{\mathrm{T}}\left[\frac{1}{2}+\frac{1}{2} \frac{1}{\left(1+8 K_{\mathrm{d}} c_{\mathrm{T}}\right)^{1 / 2}}\right] .
\end{aligned}
$$

This equation is valid also for highly ionizable surfactants such as alkyl sulfonates and sulfates $\left(K_{\mathrm{a}}>10\right)$ for $\mathrm{pH}$ values above 5 .

The adsorption density at the liquid/air interface $\left(\Gamma_{T}\right)$ has been considered to attain a constant value at concentrations as low as $1 / 3$ CMC (36). Under those conditions, Eq. [27] can be integrated to yield

$$
\begin{array}{r}
-\frac{\Gamma}{2.3 R T \Gamma_{\mathrm{T}}}=\frac{1}{2} \log \left[c_{\mathrm{T}} \frac{\left(1+8 K_{\mathrm{d}} c_{\mathrm{T}}\right)^{1 / 2}-1}{\left(1+8 K_{\mathrm{d}} c_{\mathrm{T}}\right)^{1 / 2}+1}\right] \\
+ \text { const. } \quad[28]
\end{array}
$$


Dependence of $\gamma$ on $c_{\mathrm{T}}$ under constant $\Gamma_{\mathrm{T}}$ conditions can be used to determine the value of $K_{\mathrm{d}}$.

\section{EXPERIMENTAL}

\section{Surface Tension}

Wilhelmy plate method with a sandblasted platinum plate was used in the present study to measure the surface tension.

\section{Surface Potential}

In addition to surface tension, information on surfactant adsorption at the liquid/gas interface was necessary for a quantitative analysis of the surface tension data at $\mathrm{pH}$ 11.4. This was obtained in the current study by monitoring the surface potential of the solution as a function of surfactant concentration.

Surface potential was measured by the ionization electrode technique using ${ }^{241} \mathrm{Am}$ (20 $\mu \mathrm{C})$ as air electrode and $\mathrm{Ag} / \mathrm{AgCl}$ as the reference electrode in solution. The voltage developed between the air and the reference electrode was measured using a high impedance electrometer.

Both surface potential and surface tension were measured using the same solution. In all cases, $\mathrm{pH}$ of the solutions was monitored during the surface tension measurements to ensure absence of any $\mathrm{pH}$ drift due to possible uptake of $\mathrm{CO}_{2}$ by the solution. Even though no significant drift in $\mathrm{pH}$ was observed at $\mathrm{pH}$ 11.4 , a separate series of surface tension measurements was conducted using buffered solutions.

\section{MATERIALS}

\section{Oleic Acid}

Oleic acid $(>99.9 \%$ as per the chromatographic analysis provided by the Applied Science Laboratories) in 1-g ampoules sealed under nitrogen atmosphere was stored in a refrigerator. Stock solution of potassium oleate $\left(6-7 \times 10^{-3} \mathrm{kmol} / \mathrm{m}^{3}\right)$ prepared in deaerated triple distilled water was also stored under ni- trogen atmosphere in a refrigerator. Solutions of desired oleate concentrations were prepared by diluting the stock solution just prior to the measurement.

\section{RESULTS AND DISCUSSIONS}

\section{Dependence of $\gamma$ on Total Oleate at $\mathrm{pH} 11.4$}

Results for surface tension of potassium oleate solutions as a function of concentration at pH 11.4 are shown in Fig. 2. Absence of a surface tension minimum in this curve indicates that surface active impurities are not present to any significant extent in the oleate sample. Most interestingly, the descending part of the surface tension curve is found to exhibit a decreasing slope above a certain concentration. A decrease in $\partial \gamma / \partial \log c_{\mathrm{T}}$ as a function of concentration is rather unusual and, to the author's knowledge, has not been reported in the past. This behavior of $\gamma$ vs $\log \mathcal{C}_{\mathrm{T}}$ curve is analyzed below using the expressions derived in the earlier section.

Dependence of $\gamma$ on $\log c_{\mathrm{T}}$ is governed by Eq. [27] rewritten in the following manner:

$$
-\partial \gamma /\left(2.3 R T \partial \log c_{\mathrm{T}}\right)=\Gamma_{\mathrm{T}} \alpha .
$$

According to Eq. [29] the observed decrease in $\partial \gamma / \partial \log c_{T}$ can either be due to a decrease in $\Gamma_{\mathrm{T}}$ or in $\alpha$. Since the surfactant adsorption at the liquid/air interface cannot decrease with an increase in its bulk activity, the observed decrease in $\partial \gamma / \partial \log c_{\mathrm{T}}$ is indicative of a decrease in $\alpha$.

Since $\partial \gamma / \partial \log c_{\mathrm{T}}$ is proportional to only the product of $\Gamma_{\mathrm{T}}$ and $\alpha$, determination of $\alpha$ requires a knowledge of the dependence of $\Gamma_{T}$ on oleate concentration. This was obtained in the current study from the measurements of the surface potential of the solution as a function of oleate concentration. Surface tension and surface potential results simultaneously determined for oleate solutions as a function of concentration showed that the surface potential attained a constant value at about 6 $\times 10^{-6} \mathrm{kmol} / \mathrm{m}^{3}$ of oleate (see Fig. 3), suggesting $\Gamma_{\mathrm{T}}$ to be constant in this region. Under 


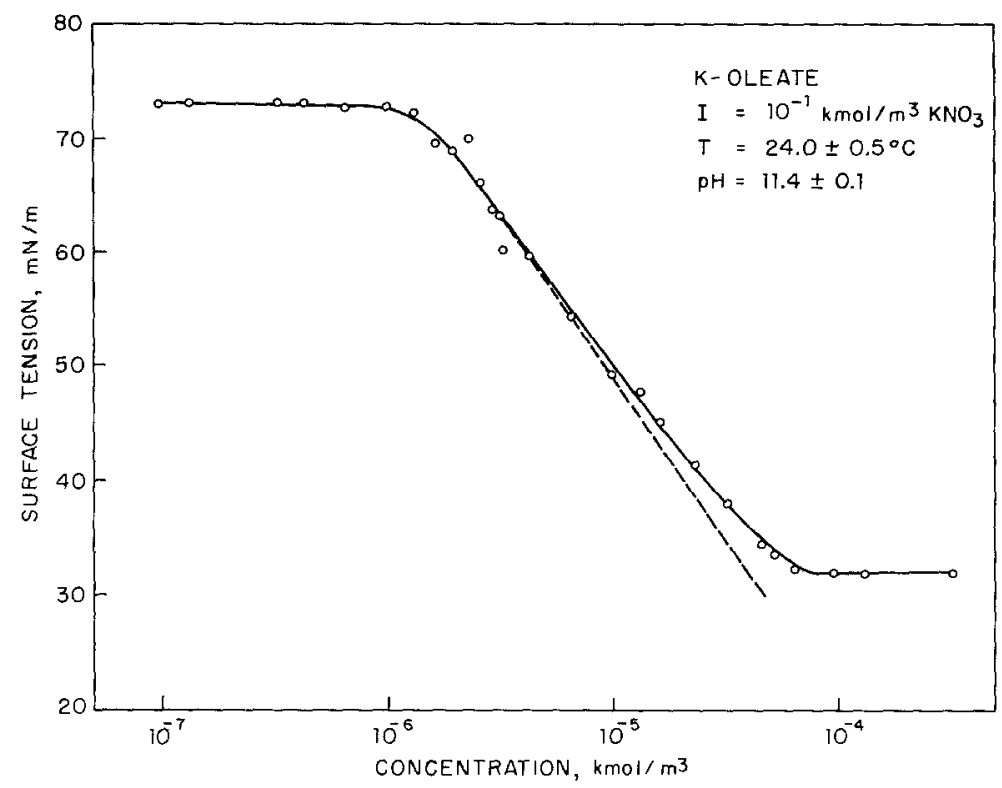

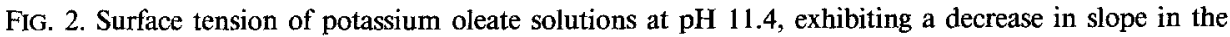
premicellar region.

conditions of constant $\Gamma_{\mathrm{T}}$, the integrated form of the following equation can be used to describe the surface tension dependence on oleate concentration:

$$
\begin{array}{r}
\frac{\gamma}{2.3 R T \Gamma_{\mathrm{T}}}=-\frac{1}{2} \log \left[c_{\mathrm{T}} \frac{\left(1+8 K_{\mathrm{d}} c_{\mathrm{T}}\right)^{1 / 2}-1}{\left(1+8 K_{\mathrm{d}} c_{\mathrm{T}}\right)^{1 / 2}+1}\right] \\
+ \text { const. } \quad[30]
\end{array}
$$

The value of $\Gamma_{\mathrm{T}}$ in the above equation can be determined from the constant slope region. Assuming different values of $K_{\mathrm{d}}$ and using the above expression, one can calculate $\gamma$ as a function of oleate concentration. Calculated values of $\gamma$ for different values of $K_{\mathrm{d}}$ are plotted in Fig. 4. It is clear from this figure that the calculated dependence of $\gamma$ on total oleate concentration is in agreement with the experimentally obtained $\gamma$ vs $\log c_{\mathrm{T}}$ curve for $K_{\mathrm{d}}=6 \times 10^{3}\left(\mathrm{kmol} / \mathrm{m}^{3}\right)^{-1}$.

The above value of $K_{\mathrm{d}}, 6 \times 10^{3}$, was obtained from the $\gamma$ vs $\log c_{\mathrm{T}}$ curve of an unbuffered system. Since small changes in $\mathrm{pH}$ can significantly influence the surface tension of oleate solutions, the data for dependence of $\gamma$ vs $\log c_{\mathrm{T}}$ was obtained in the presence of
$\mathrm{KOH} / \mathrm{K}_{2} \mathrm{HPO}_{4}$ buffer (see Fig. 3). These results yielded a value of $K_{\mathrm{d}}$ equal to $5 \times 10^{3}$, which is in close agreement with the value obtained in the absence of the buffer.

\section{Comparison of the Present Results with the Literature Values of $K_{\mathrm{d}}$ for Oleate Dimer}

The value of $K_{\mathrm{d}}$ obtained from the present study $\left(5-6 \times 10^{3}\left(\mathrm{kmol} / \mathrm{m}^{3}\right)^{-1}\right)$ is in agreement

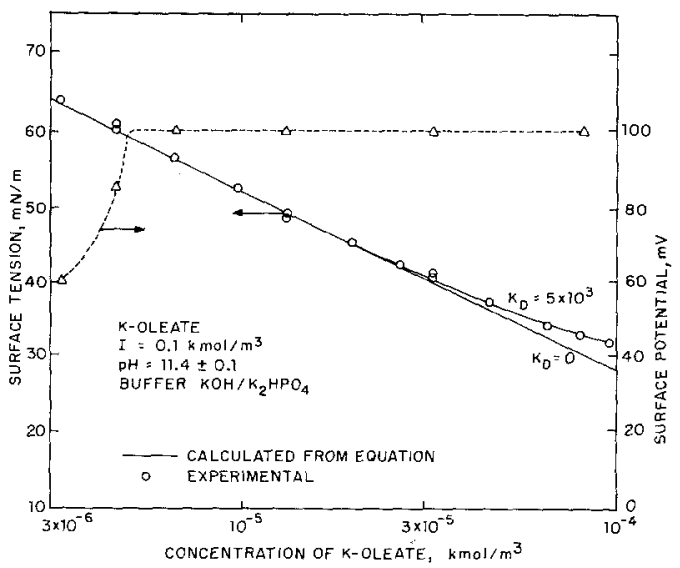

FIG. 3. Surface tension and surface potential of potassium oleate solutions at $\mathrm{pH} 11.4$ as a function of total oleate concentration. 


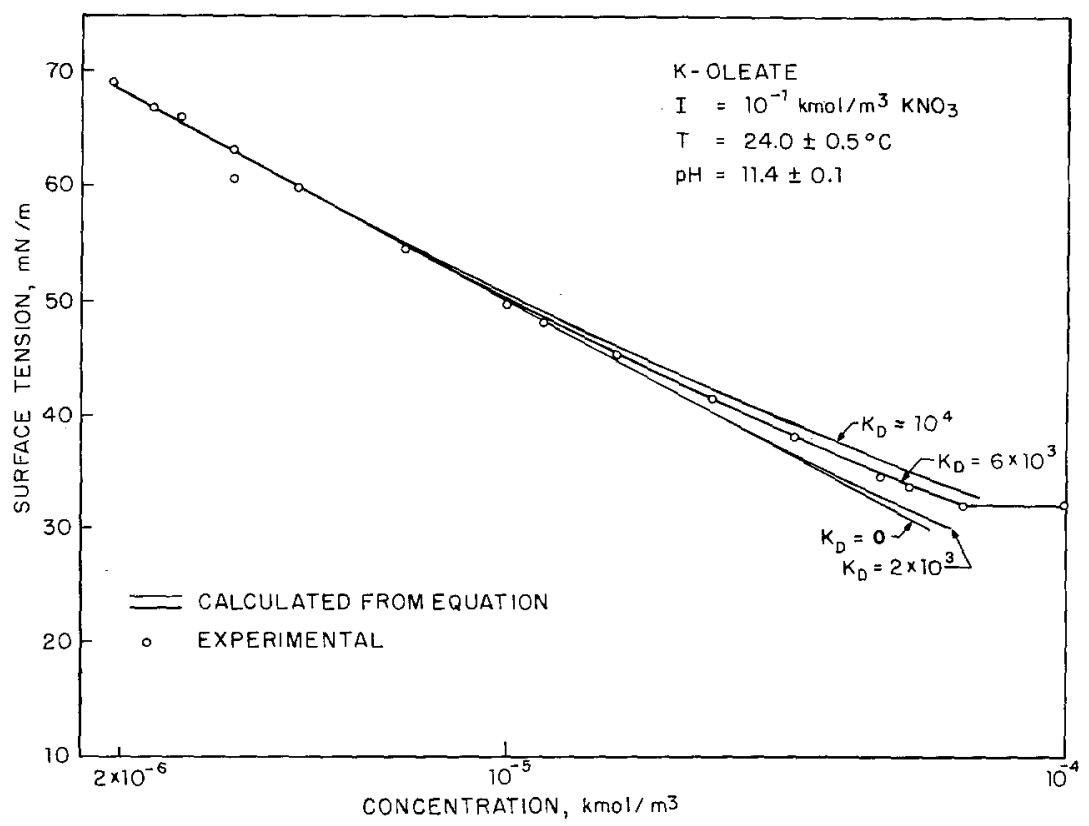

FIG. 4. Correlation of the calculated values of surface tension with the experimental data.

with the value of $10^{4}\left(\mathrm{kmol} / \mathrm{m}^{3}\right)^{-1}$ reported by Jung (20). The latter value was obtained by assuming a linear dependence for $\log$ (solubility) vs chain length and by attributing the deviations in $\log$ (solubility) from the above behavior to dimerization in the aqueous phase. The present value as well as the one reported by Jung (20) is, however, found to differ significantly from the value $7.2 \times 10^{6} \mathrm{(kmol} /$ $\left.\mathrm{m}^{3}\right)^{-1}$ reported by Mukerjee (11). The latter value was obtained from literature data for the partitioning of oleic acid between heptane and aqueous phase at $\mathrm{pH} 7.45$. Under neutral $\mathrm{pH}$ conditions, formation of oleate-oleic acidsoap can be expected and this would indeed result in high values of $K_{\mathrm{d}}$. In fact our estimates of $K_{\mathrm{ad}}$ based upon surface tension measurements at $\mathrm{pH} 9.4$ has yielded a value of 1.3 $\times 10^{7}$ and this possibly suggests that the value reported by Mukerjee may correspond to that for acid-soap formation. Furthermore, as pointed out by Mukerjee (11), the uncertainties in the constants for aggregation in the heptane phase makes the estimates of $K_{\mathrm{d}}$ in the aqueous phase obtained from partitioning data less reliable. In contrast to this, the experimental conditions selected during the present study ( $\mathrm{pH}$ 11.4) is free from such complications due to acid-soap formation and consequently makes the present estimate of $K_{\mathrm{d}}$ more reliable.

\section{SUMMARY}

The formation of premicellar aggregates such as dimers in aqueous potassium oleate solutions was investigated by measuring the surface tension of oleate solutions under high $\mathrm{pH}(\cong 11.4)$ conditions. Results have been analyzed using a thermodynamic model which takes into account the formation of dimers and acid-soaps. The observed decrease in $\partial \gamma /$ $\partial \log c_{\mathrm{T}}$ above a certain concentration (below $\mathrm{CMC}$ ) has been attributed to the formation of doubly charged dimers and the formation constant for oleate dimer has been estimated to be $6 \times 10^{3}\left(\mathrm{kmol} / \mathrm{m}^{3}\right)^{-1}$.

\section{ACKNOWLEDGMENTS}

Support of the Department of Energy (DE-AC19-79BC10082), The National Science Foundation (CPE-8201216), Amoco Production Company, Chevron Oil Field Research Company, Exxon Research and Engineering 
Company, Gulf Research and Development Company, Marathon Oil Company, Shell Development Company, Sohio, Texaco Inc., and Union Oil Company of California is gratefully acknowledged.

\section{REFERENCES}

1. McBain, J. W., Kolloid Z. 12, 256 (1913).

2. McBain, J. W., and McHan, H., J. Amer. Chem. Soc. 92, 141 (1940).

3. Ekwall, P., Kolloid Z. 92, 141 (1940).

4. Ekwall, P., and Lindblad, L. G., Kolloid Z. 94, 42 (1941).

5. Stauff, J., Physik Chem. 183A, 55 (1938).

6. Cook, M. A., J. Phys. Colloid Chem. 55, 383 (1951).

7. Mukerjee, P., Mysells, K. J., and Dulin, C. I., J. Phys. Chem. 62, 1390 (1958).

8. Mukerjee, P., J. Phys. Chem. 62, 1397 (1958).

9. Mukerjee, P., and Mysels, K. J., J. Phys. Chem. 62, 1400 (1958).

10. Mukerjee, P., J. Phys. Chem. 62, 1404 (1958).

11. Mukerjee, P., J. Phys. Chem. 69, 2821 (1965).

12. Eagland, D., and Franks, F., Trans. Faraday Soc. 61, 2468 (1965).

13. Franks, F., and Smith, H. T., Trans. Faraday Soc. 63, 2585 (1967).

14. Franks, F., and Smith, H. T., J. Phys. Chem. 68, 3581 (1964).

15. Franks, F., Quickender, M. J., Ravenhill, J. R., and Smith, H. T., J. Phys. Chem. 72, 2668 (1968).

16. Bangs, L. B., "Surfactant Dimers and Their Adsorption," Ph.D. Thesis, MTT, Cambridge, Mass., 1964.

17. Walton, H. F., Heibert, E. N., and Scholtes, E. H., J. Colloid Sci. 1, 385 (1946).

18. Proust, J., and Ter-Minassian, L. S., C. R. Acad. Sci., Paris 270C, 1354 (1970). (French text.)

19. Mukerjee, P., Advan. Colloid Interface Sci., 1, 241 (1967).
20. Jung, R. F., "Oleic Acid Adsorption at the Goethite Water Interface", M.S. Thesis, Univ. of Melbourne, 1976.

21. Lucassen, J., J. Phys. Chem. 70, 1824 (1966).

22. Eagland, D., and Franks, F., Preassociation and thermodynamics of hydrophobic hydration in dilute solutions of surfactants, Proc. Int. Congr. Surface Active Substances, Brussels, Sept. 1964.

23. Goodman, D. W., J. Amer. Chem. Soc. 80, 3887 (1958).

24. Frank, H. S., and Evans, M. W., J. Phys. Chem. 13, 507 (1945).

25. Nemethy, G., and Scheraga, H. A., J. Phys. Chem. 66, 1773 (1962).

26. Nemethy, G., and Scheraga, H. A., J. Chem. Phys. 36, 3401 (1962).

27. Schrier, E. E., Pottle, M., and Scheraga, H. A., $J$. Amer. Chem. Soc. 86, 3444 (1964).

28. Parfitt, G. D., and Smith, A. L., J. Phys. Chem. 66, 942, 1962.

29. Van Voorst Vader, F., Trans. Faraday Soc. 57, 110 (1961).

30. Van Voorst Vader, F., Trans. Faraday Soc. 56, 1067 (1960).

31. Kolthoff, 1. M., and Johnson, W. F., J. Phys. Colloid Chem. 52, 22 (1948).

32. Malik, W. V., Srivastava, S. K., and Gupta, D., J. Electroanal. Chem. 34, 540 (1972).

33. Rijnbout, J. B., J. Coll. Interface Sci. 62, 81 (1977).

34. Finch, J. A., and Smith, G. W., J. Appl. Chem. Biotechnol. 25, 271 (1975).

35. Ananthapadmanabhan, K. P., "Associative Interactions in Surfactant Solutions and Their Role in Flotation," D.E.Sc. Thesis, Columbia Univ., New York, 1980.

36. Rosen, M. J., "Surfactants and Interfacial Phenomena." Wiley, New York, 1978. 